\title{
Association of Blood Groups with the Severity and Outcome of COVID-19 Infection in Children
}

\author{
Attia Bari ${ }^{1}$, Aimen $\mathrm{Ch}^{1}$, Sidra Hareem ${ }^{2}$, Iqbal Bano ${ }^{1}$, Junaid Rashid ${ }^{1}$ and Masood Sadiq ${ }^{3}$ \\ ${ }^{1}$ Department of Pediatric Medicine, The Children's Hospital and The Institute of Child Health, Lahore, Pakistan \\ ${ }^{2}$ Department of Pediatric Hematology, The Children's Hospital and The Institute of Child Health, Lahore, Pakistan \\ ${ }^{3}$ Department of Pediatric Cardiology, The Children's Hospital and The Institute of Child Health, Lahore, Pakistan
}

\begin{abstract}
The objective of this study was to find out the association of $A B O$ blood groups with the severity and outcome of corona virus disease 2019 (COVID-19) in children. It included all laboratory-confirmed cases of COVID-19 and post-COVID multisystem inflammatory syndrome in children (MIS-C)/ Kawasaki disease (KD) like illness, admitted from March to September, 2020 to The Children's Hospital, Lahore. Out of 66 children, 45 (68.2\%) were COVID-19 and 21 (31.8\%) MIS-C/KD temporally associated with SARS-COV-2. The mean age was $7.9 \pm 4.2$ years. Majority of children had mild to moderate illness $38(57.6 \%)$, while $23(34.8 \%)$ had severe or critical disease. Among all patients, 24 (36.4\%) had some underlying comorbidity. Blood group A was significantly associated with severe and critical disease $(p=0.030)$. COVID-19 in children had generally a good outcome, but children with blood group A were more susceptible to severe/critical disease.
\end{abstract}

Key Words: Coronavirus disease 2019, ABO blood groups, Children, Severity, Outcome.

How to cite this article: Bari A, Ch A, Hareem S, Bano I, Rashid J, Sadiq M. Association of Blood Groups with the Severity and Outcome of COVID-19 Infection in Children. J Coll Physicians Surg Pak 2021; 31(JCPSPCR):CR57-CR59.

\section{INTRODUCTION}

Corona virus disease 2019 (COVID-19), caused by a novel coronavirus (SARS-CoV-2), resulted in global pandemic after a severe outbreak in China. The World Health Organisation (WHO) declared COVID-19 as a Global health emergency and a pandemic disease on $11^{\text {th }}$ March, 2020. COVID-19 is generally a milder disease in children, but severe cases have been reported in the literature. ${ }^{1} \mathrm{Few}$ children present with hyper-inflammatory condition called as multisystem inflammatory syndrome in children (MIS-C) or Kawasakidisease (KD) like illness. ${ }^{2}$

Researches on COVID-19 patients described a relationship between $\mathrm{ABO}$ blood groups and severity of viral infection. There is a differential susceptibility of personstowards COVID-19 infection in various blood groups as the human blood type is noted to be a biomarker of susceptibility of COVID-19 infection; and the people with blood group A may need particularly augmented personal protection to reduce the chance of infection and to receive more cautious surveillance and aggressive management. $^{3}$

Correspondence to: Dr. Attia Bari, Department of Pediatric Medicine, The Children's Hospital and The Institute of Child Health, Lahore, Pakistan

E-mail:drattiabari@gmail.com

Received: November 20, 2020; Revised: February 02, 2021; Accepted: February 16, 2021

DOI: https://doi.org/10.29271/jcpsp.2021.JCPSPCR.CR57
The published data on COVID-19 in children primarily comes from China, USA and Europe. Limited data is available from the developing world and the published literature from the developing world is also lacking with special reference to blood groups in COVID-19. This study analysed the disease severity of COVID-19 and post-COVID MIS-C/KD in children and association of blood groups with the severity and outcome of disease presenting to a dedicated pediatric hospital.

After taking IRB approval and informed consent from the parents, this descriptive cross-sectional study was conducted from March to September 2020 at The Children's Hospital and Institute of Child Health, Lahore.

All confirmed children with COVID-19, based on positive COVID-19PCR, and MIS-C/KD based on positive serology for antibody, were included in the study. Blood grouping was done in all patients in the hospital blood bank. The disease severity was categorised as asymptomatic, mild, moderate, severe, and critical; and the diagnosis of post-COVID MIS-C/ KD was made as per WHO criteria.

The information collected was analysed by using statistical software SPSS-22. The quantitative variables like age and duration of hospital stay is presented as mean and SD. Qualitative variables like blood group, disease severity and outcome are presented by calculating frequency and percentages. Chisquare test or Fisher's Exact test was employed for categorical variables and Independent sample t-test for continuous variable. The $p$-value $<0.05$ was considered as significant. 


\begin{tabular}{|c|c|c|c|c|c|c|}
\hline \multicolumn{7}{|l|}{ ABO blood groups } \\
\hline Variables & Total & $\mathbf{A}$ & B & $\mathbf{A B}$ & 0 & p-value \\
\hline \multicolumn{7}{|l|}{ Disease severity } \\
\hline Asymptomatic & 5 & $1(20)$ & $1(20)$ & $0(0)$ & $3(60)$ & 0.030 \\
\hline Mild- Moderate & 38 & $4(10.5)$ & $14(36.8)$ & $1(2.6)$ & $19(50)$ & \\
\hline Severe-Critical & 23 & $12(52.2)$ & $8(34.8)$ & $0(0)$ & $3(13)$ & \\
\hline \multicolumn{7}{|l|}{ Respiratory support } \\
\hline None & 40 & $5(12.5)$ & $14(35)$ & $0(0)$ & $21(52.5)$ & 0.014 \\
\hline Oxygen & 23 & $11(47.8)$ & $7(30.4)$ & $1(4.3)$ & $4(17.4)$ & \\
\hline Mechanical ventilation & 3 & $1(33.3)$ & $2(66.7)$ & $0(0)$ & $0(0)$ & \\
\hline \multicolumn{7}{|l|}{ ICU/HDU care } \\
\hline None & 39 & $5(12.8)$ & $13(33.3)$ & $0(0)$ & $21(53.8)$ & 0.006 \\
\hline $\mathrm{ICU}$ & 12 & $4(33.3)$ & $6(50)$ & $0(0)$ & $2(16.7)$ & \\
\hline HDU & 15 & $8(53.3)$ & $4(26.7)$ & $1(6.7)$ & $2(13.3)$ & \\
\hline \multicolumn{7}{|l|}{ Outcome } \\
\hline Discharged & 61 & $15(24.6)$ & $20(32.8)$ & $1(1.6)$ & $25(41)$ & 0.316 \\
\hline Expired & 5 & $2(40)$ & $3(60)$ & $0(0)$ & $0(0)$ & \\
\hline
\end{tabular}

A total of 74 children with confirmed COVID-19 and MIS-C were admitted over the study period. Eight asymptomatic/mild disease children in whom blood group was not done, were excluded. The mean age of the remaining 66 children was $7.9 \pm 4.2$ years with a male preponderance $38 / 66$ (57.6\%). There was no significant difference in the mean age of children with COVID-19 and MIS-C/KD $(8.1 \pm 4.6$ vs. $7.5 \pm$ 3.3 years) $(p=0.538)$. Blood type $O$ was the predominant blood group as 25 (37.9), and $A B$ was found in only one child. Majority of children had mild to moderate illness 38 $(57.6 \%), 23(34.8 \%)$ had severe or critical disease, and 05 $(7.6 \%)$ were asymptomatic. In the initial phase of this pandemic, all patients who tested positive were being isolated in hospitals till they tested negative.

Blood group A was significantly associated with severe and critical disease $(p=0.030$, Table-l), respiratory support requirement and need for ICU admission $(p=0.014)$ and $(p=0.006)$, respectively.

There was no statistical difference in the blood group between COVID-19 and post-COVID MIS-C/ KD $(p=0.109)$ and presence of underlying comorbidity $(p=0.064)$.

Underlying comorbidity was present in 24 (36.4\%), chronic kidney disease being the most common followed by congenital heart disease and surgical cases. The case fatality rate was $5(7.6 \%)$ and all deaths were due to underlying comorbid conditions; and one death was due to MIS-C, in which there was delayed diagnosis. There was no significant relationship of comorbid conditions and the blood group (0.316).

Since the recent widely reported literature on possible association between blood groups and COVID-19, there is concern about COVID-19 and specific blood groups. It is also documented that there might be a prognostic significance of blood type in COVID-19 and is related to disease severity and outcome. The researchers from around the world found a link between more severity of COVID-19 in type A blood group, while blood group $\mathrm{O}$ was associated with a less severe disease. ${ }^{4}$ There is no published literature on this aspect in children with COVD-19. Blood group 0 was the commonest blood group found in this study, followed by $B$ and $A$. In a study from Pakistan, blood group A was documented to be the commonest blood group found in $37.4 \%$ of patients with COVID-19, followed by blood group B; and in general population the most prevalent blood group was $\mathrm{B}^{5}$

In this study, only 04 (6.1\%) children had Rh negative blood group when analysed on the basis of Rh blood group; and similar findings were reported in a research from Turkey that $\mathrm{Rh}$ positive blood group was significantly higher in COVID-19 $(p=<0.001){ }^{6}$ In this study, all four children with Rh-negative blood type had mild to moderate illness and no death was reported. The plausible explanation is that negative blood group is protective and the Rh-positive blood group people are predisposed to COVID-19.

This was a single-centre study limiting the generalisation of the present results. However, the Children's Hospital, Lahore is the largest referral centre for children in Pakistan which provides a comprehensive picture of pediatric patients suffering from COVID-19 in Pakistan with varying degree of disease severity. The current study suggests that the most prevalent blood group in children admitted with COVID-19 infection was blood group 0, but these children had milder disease. Moreover, the children having blood group A were more susceptible to severe or critical disease. COVID-19 in children has generally a good outcome.

This study provides important data on COVID-19 in children and the association of $A B O$ blood groups with disease severity from a developing country.

\section{ETHICAL APPROVAL:}

Institutional Review Board has approved the study for publication.

\section{CONFLICT OF INTEREST:}

The authors declared no conflict of interest.

\section{AUTHORS' CONTRIBUTION:}

$A B$ : Conceived, designed, manuscript writing. 


\section{AC: Data collection.}

SH: Lab data collection.

IB: Proofreading.

JR: Intellectual input.

MS: Intellectual input \& final approval.

\section{REFERENCES}

1. Hong H, Wang Y, Chung HT, Chen CJ. Clinical characteristics of novel coronavirus disease 2019 (COVID-19) in newborns, infants and children. Pediatr Neonatol 2020; 61(2):131-2.

2. Sadiq M, Aziz OA, Kazmi U, Hyder N, Sarwar M, Sultana N, et al. Multisystem inflammatory syndrome associated with COVID-19 in children in Pakistan. Lancet Child Adolesc Health 2020; 4(10): e36-e37. doi:10.1016/S2352-4642
(20)30256-X.

3. Zhao J, Yang Y, Huang HP, Li D, Gu DF, Lu XF, et al. Relationship between the ABO Blood Group and the COVID-19 Susceptibility. Clin Infect Dis 2020; Ciaa 1150. doi: 10.1093/$\mathrm{cid} / \mathrm{ciaa} 1150$.

4. Rubin R. Investigating whether blood type is linked to COVID-19 Risk. JAMA 2020; 324(13):1273. doi: 10.1001/ jama.2020.16516.

5. Noor A, Tashfeen S, Fahim AN, Din HU, Akhtar A. Association of COVID-19 with ABO blood groups in tertiary care center of Pakistan. Pak Armed Forces Med J 2020; 70(1):251-5.

6. Arac E, Solmaz I, Akkoc H, Donmezdil S. Association between the rh blood group and the Covid-19 susceptibility $\mathrm{i}$ nternational journal of $\mathrm{h}$ ematology and o ncology association between the rh blood group and the Covid-19 Susceptibility. Int J Hemat Oncol 2020; 2(30):81-6. 